\title{
Estudio exploratorio de valores hematológicos en terneras Holstein Frisian mestizas, durante los primeros seis meses de vida
}

\section{Palacios, T.E., Narváez, J.A.}

Carrera Medicina Veterinaria y Zootecnia, Facultad de Ciencias Agropecuarias, Universidad de Cuenca, Cuenca, Ecuador.

Autor para correspondencia: estuardo.palacios@ucuenca.edu.ec

Fecha de recepción: 16 de octubre 2017 - Fecha de aceptación: 28 de mayo 2018

\section{RESUMEN}

Los cambios relacionados con la edad en los valores hematológicos se producen en casi todas las especies de mamíferos, y los estudios publicados que incluyen mediciones repetidas de los parámetros hematológicos en terneras durante los primeros meses de vida son limitados. El objetivo de la presente investigación fue determinar los valores hematológicos con mediciones secuenciales, desde el nacimiento hasta los 6 meses de edad, en terneras de la raza Holstein frisian mestizas, en la granja de Nero ubicada a 3,000 msnm. Se tomaron muestras de sangre a 36 terneras clínicamente sanas, las pruebas hematológicas se realizaron en contadores celulares automáticos de uso veterinario y calibrado para la especie bovina. Los resultados fueron procesados mediante el software SPSS versión 22, en él se calcularon los valores descriptivos mínimos, máximos, el promedio y la desviación estándar para la edad en días y los valores hematológicos. Además de los estadísticos descriptivos, se aplicó la prueba de Correlación de Pearson para establecer si existe correlación entre los valores evaluados con respecto a la edad. Los niveles de significancia se establecieron a dos colas tanto para $\mathrm{p} \leq 0.01(* *)$ como para $\mathrm{p} \leq 0.05(*)$. Como conclusión se puede manifestar que la mayoría de las correlaciones evidenciadas presenta una asociación baja con la edad, sin embargo, el $\mathrm{VCM}^{1}$ tiene una asociación media (Correlación de Pearson $-0.531^{* *}, \mathrm{R}^{2}=0.28$ ), misma que disminuye a medida que aumenta la edad de las terneras.

Palabras clave: Terneras, valores hematológicos, Holstein Frisian.

\author{
ABSTRACT

\footnotetext{
1 Abreviaturas y unidades:

RBC $10^{12} / \mathrm{L}$ : glóbulos rojos $\left(10^{12} / \mathrm{L}\right)$

HGB g/L: hemoglobina (g/L)

HCT \%: hematocrito (\%),

VCM fL: volumen corpuscular medio (fentolitro o fL)

HCM pg: hemoglobina corpuscular media (picogramo o pg)

CHCM g/L: concentración de hemoglobina corpuscular media en g/100 ml

WBC $10^{9} / \mathrm{L}$ : glóbulos blancos $\left(10^{9} / \mathrm{L}\right)$

LYM \% o $10^{9} / \mathrm{L}$ : linfocitos (\% o $\left.10^{9} / \mathrm{L}\right)$

MID $\%$ o $10^{9} / \mathrm{L}:$ monocitos $\left(\%\right.$ o $\left.10^{9} / \mathrm{L}\right)$

GRAN \% o $10^{9} / \mathrm{L}$ : neutrófilos, basófilos, eosinófilos (GRAN) \% o 10\%/L

PLT $10^{9} / \mathrm{L}$ : plaquetas $\left(10^{9} / \mathrm{L}\right)$
}

Age-related changes in hematological values occur in almost all mammalian species, and published studies that include repeated measurements of hematological parameters in calves during the first months of life are limited. The objective of the present investigation was to determine the hematological values with sequential measurements from birth to 6 months of age in calves of the Holstein breed Frisian mestizas, in the Nero farm located at 3000 masl. Blood samples were taken from 36 clinically healthy heifers, hematological tests were performed on automatic cell counters for veterinary use and calibrated for the bovine species. The results were processed using the software SPSS version 22, in 
which the minimum, maximum, average and the standard deviation for the age in days and the hematological values were calculated. In addition, from the descriptive statistics the Pearson Correlation test was applied to establish whether there is a correlation between the evaluated values with respect to age. The significance levels were established to two tails for both $\mathrm{p} \leq 0.01(* *)$ and $\mathrm{p} \leq 0.05(*)$. In conclusion, it can be stated that most correlations evidenced have a low association with age, however, the $\mathrm{MCV}^{1}$ has an average association (Pearson Correlation $-0.531^{* *}, \mathrm{R}^{2}=0.28$ ), which decreases to as the age of calves increases.

Keywords: Calves, hematological values, Frisian Holstein.

\section{INTRODUCCIÓN}

En terneros las irregularidades metabólicas son alteraciones que se presentan con mayor frecuencia en forma subclínica, donde el desarrollo se detiene y no existe un incremento de peso, por lo tanto, el diagnóstico se dificulta debido a que las apariencias manifiestan buen estado de salud sin que el propietario y el médico veterinario perciban de su presencia. Con estas evidencias y con el avance de la tecnología en el campo de la medicina veterinaria, es menester determinar los cambios ontogénicos de algunos parámetros hemáticos de interés diagnóstico que pudieran ocurrir en terneras, desde su nacimiento hasta los 6 meses de vida, y, a la vez, tener intervalos de referencia específicos para cada especie animal para una interpretación adecuada de los resultados de los análisis hematológicos y bioquímicos, que a la vez sirvan como una herramienta de toma de decisiones médicas. Mohri, Sharifi, \& Eidi (2007) señalaron que parámetros hematológicos tales como hemoglobina (HGB), VCM, HCM, CHCM y neutrófilos en los tres primeros meses de vida, deben ser considerados para la precisa interpretación de los resultados de laboratorio. De igual manera se debe considerar que el perfil hematológico es un examen paraclínico, empleado en el diagnóstico de las enfermedades metabólicas, el cual se debe comparar con valores de referencia según la especie, edad y estado fisiológico. Roldán et al. (2005) y Ježek, Nemec, Starič, \& Klinkon (2011) señalaron que los valores de referencia de diferentes variables sanguíneas están bien establecidos para el ganado adulto, pero para los terneros no hay muchos datos disponibles; además, en los terneros, están cambiando con la edad y los valores de los analitos cambian después del nacimiento debido a la ingesta de calostro, el tiempo de vida corto de los eritrocitos, y la disminución de la concentración de hemoglobina fetal. De igual forma, en los terneros en crecimiento, el sistema de alimentación y crianza tiene una influencia importante en los valores de las variables hematológicas. Sandoval et al. (2007), citado por Delgado, Rodríguez, Barreto, \& Vazquez (2014), indicaron que los indicadores hematológicos constituyen un examen paraclínico que permite conocer la relación entre los desórdenes en la salud y las deficiencias nutricionales.

Así mismo, debemos considerar que los intervalos de referencia comúnmente utilizados para el ganado se basan generalmente en muestras obtenidas de animales adultos y pueden ser engañosas si se utilizan para caracterizar valores de individuos jóvenes, a la vez, las diferentes metodologías analíticas pueden interferir en los intervalos de referencia (Brun-Hansen, Kampen, \& Lund, 2006). En la actualidad la información sobre los intervalos de referencia en terneras a diferentes edades a nivel mundial es limitada, y con relación a nivel nacional y regional es escasa, apenas se conoce de este tipo de estudios y, los pocos existentes, son de mucho tiempo atrás, además, cabe indicar que los métodos utilizados en estudios previos nacionales, fueron manuales y en la actualidad se emplea la tecnología automática, que brinda resultados más reales y confiables. Al no existir estudios que incluyan mediciones repetidas de los parámetros hematológicos en terneras durante los primeros meses de vida, en zonas de altitudes que oscilan entre los 2,800 a 3,200 msnm, es de suma importancia la realización de este estudio y así obtener valores hematológicos de referencia que nos permitan ayudar al diagnóstico de patologías que estén relacionadas con el futuro de la producción y reproducción de los bovinos. Más aun, tomando en consideración que la provincia del Azuay está en el ranking de las zonas de mayor producción lechera en el país, según datos de Agrocalidad $^{2}$.

\footnotetext{
${ }^{1}$ MCV: Medium Corpuscular Volumen

${ }^{2}$ Agrocalidad: www.agrocalidad.gob.ec
} 
En este sentido, el hemograma es un estudio que nos brinda información cualitativa y cuantitativa; siendo la base fundamental del estudio clínico-patológico del paciente, dada su capacidad de reflejar un sinnúmero de situaciones y esclarecer numerosas interrelaciones; además el hemograma adquiere relevancia para el diagnóstico, pronóstico y monitoreo de la terapia (Coppo, 2010). De igual manera, el médico veterinario debe conocer las funciones y alteraciones del sistema cardiovascular, ya que éste es esencial para la vida y la salud del animal, tomando en cuenta que los componentes celulares de la sangre incluyen los glóbulos rojos o eritrocitos, glóbulos blancos o leucocitos y plaquetas o trombocitos (Klein, 2014). Kasai (2006), citado por Coppo \& Mussart (2006), manifiesta que el hemograma sigue constituyendo el pilar fundamental de las exploraciones de laboratorio, a tal punto que actualmente es utilizado para verificar la normalidad de terneros nacidos de hembras clonadas.

La función principal del sistema circulatorio es el transporte de sangre para suministrar oxígeno a todos los tejidos del cuerpo, así como la remoción de bióxido de carbono y bioproductos del metabolismo celular de los mismos (Saíz, Antuna Bizarro, \& Anzaldúa Arce, 2010). Los índices de los glóbulos rojos, que nos ayudan a determinar ciertos procesos patológicos relacionados a las anemias son: VCM, HCM y CHCM (Latimer, Mahaffey, \& Prasse, 2005). En el recuento de leucocitos se pueden presentar variaciones fisiológicas como consecuencia de estrés, ejercicio, alimentación, edad, raza y el manejo del animal al momento de la toma de muestras para análisis. Según Paez, Campos, \& Patiño (2013) los cambios en los recuentos totales de leucocitos sólo son significativos cuando presentan altas desviaciones en relación con los valores normales de referencia; cuando esto ocurre se debe sospechar la presencia de un proceso patológico. Las plaquetas sanguíneas de los mamíferos se producen a partir de los megacariocitos en la médula ósea, los mismos que se denominan UFR-megacariocito y tienen estimulación apropiada para su mitosis (Meyer \& Harvey, 2000). Las plaquetas son necesarias en la vida animal, ya que, al cumplir su función hemostática, como la formación del tapón plaquetario en el sitio de la lesión vascular, ayuda al control de las hemorragias y además colabora con el mantenimiento de la integridad vascular normal.

De acuerdo con estos antecedentes, el objetivo del presente estudio fue realizar un estudio exploratorio de los valores hematológicos en terneras con mediciones secuenciales, desde el nacimiento hasta los 6 meses de edad, tomando en consideración el aspecto fisiológico que señala Reece (2004), que durante el desarrollo fetal inicial el saco vitelino se encarga de producir glóbulos rojos nucleados, según avanza el desarrollo intervienen el hígado, bazo y nódulos linfáticos, y, en las últimas etapas de gestación y tras el nacimiento la eritropoyesis, se localiza en la medula ósea, además, en los adultos, la médula de los huesos largos son reemplazados por tejido graso, por lo tanto, según avanza la edad, la actividad medular va perdiendo su actividad de producir de glóbulos rojos.

\section{MATERIALES Y MÉTODOS}

El estudio se realizó en la granja de Nero, propiedad de la Universidad de Cuenca, ubicada a una altitud entre los 2,800 y $3,200 \mathrm{msnm}$, con una temperatura promedio de 8 a $10^{\circ} \mathrm{C}$ y una pluviosidad anual de 800 a 1,200 mm, pertenece a la parroquia rural de Baños, cantón Cuenca, provincia del Azuay. La población en estudio estuvo conformada por treinta y seis (36) terneras, y, como factor de inclusión, se consideró a todas las terneras que hayan nacido por parto normal y que sus constantes fisiológicas estén dentro de los parámetros normales, de acuerdo su edad y época de nacimiento; como factores de exclusión se consideró a las terneras que no estén dentro de la raza en estudio y terneras con problemas fisiológicos al momento del nacimiento. Las terneras fueron alimentadas con calostro durante los primeros tres días y posterior a ello con leche entera, acceso a concentrado, agua ad libitum y a potrero asignados para ellas durante el tiempo del estudio, de acuerdo con las recomendaciones específicas de la edad.

A las terneras seleccionadas se realizó la valoración clínica antes de cada muestreo y se procedió a tomar asépticamente una muestra de sangre por venipunción de la vena yugular, utilizando tubos de sangría tipo vacutainer con EDTA como anticoagulante. El monitoreo secuencial se realizó cada 15 quince días hasta que las terneras lleguen a tener la edad de dos meses, y, luego, muestreos mensuales hasta que lleguen a cumplir los seis meses o 180 días de edad. Esto está en línea con la recomendación 
de Mohri et al. (2007) quienes señalaron que parámetros hematológicos tales como HGB, VCM, HCM, CHCM y neutrófilos en los tres primeros meses de vida, deben ser considerados para la precisa interpretación de los resultados de laboratorio. Además, Ježek et al. (2011) señalaron que, en los terneros, los valores están cambiando con la edad y después del nacimiento debido a la ingesta de calostro, el tiempo de vida corto de los eritrocitos y la disminución de la concentración de hemoglobina fetal.

Los análisis de laboratorio se realizaron en un analizador hematológico automático de uso veterinario y con calibración para cada especie animal (Count cell 1800 Piruvet), de acuerdo a lo que recomineda Coppo (2010), Latimer et al. (2005), Meyer \& Harvey (2000) y Nuñez \& Bouda (2007), ya que estos equipos dan los valores más exactos, siempre que se hayan calibrado para adecuarse a la sangre de la especie a examinar; los errores metodológicos por el trabajo manual van del 2 al $8 \%$.

Antes de realizar las lecturas en el analizador automático, la sangre se homogenizó en inversión y se mantuvo durante todo el procedimiento en el homogenizador automático y se comprobó que no existan coágulos, ya que ello da lugar a que los recuentos de plaquetas y leucocitos sean falsos positivos, es decir, falsamente bajos, y así, el valor de los eritrocitos puede aumentar o disminuir (Villiers \& Blackwood, 2013). Los parámetros que se determinaron fueron: RBC, HGB y HCT; los índices eritrocíticos: VCM, HCM, CHCM; WBC y los índices leucocitarios: LYM, MID, GRAN y PLT (Meyer \& Harvey, 2000; Brun-Hansen et al., 2006).

En la presente investigación, los resultados fueron procesados mediante el Software SPSS 22, en dónde se calcularon los valores descriptivos mínimos, máximos, media y la desviación estándar para la edad en días y los valores hematológicos. Además de los estadísticos descriptivos, se aplicó la prueba de Correlación de Pearson para establecer si existe correlación entre los valores evaluados por el laboratorio con respecto a la edad. Los niveles de significancia se establecieron a dos colas tanto para $0.01(* *)$ como para $0.05(*)$.

\section{Limitaciones}

No se pudieron realizar las mediciones de los 36 animales en fechas similares cada 15 días, debido a los nacimientos que se producían en diferentes fechas. Además, se podría tener un control sobre otras variables como peso y raza. Incluso se podría incursionar en el estudio de valores hematológicos de acuerdo con las zonas como Costa, Sierra y Amazonía, en sus diferentes pisos altitudinales, y también relacionar con los datos obtenidos y con referencias para animales adultos, con la finalidad de conseguir lo que señala Mohri et al. (2007).

\section{RESULTADOS Y DISCUSIÓN}

En el presente estudio, se investigaron los valores hematológicos en 36 terneras clínicamente sanas durante los primeros 6 meses de vida. Los resultados confirmaron que varios valores hematológicos en las terneras difieren de los adultos, debido al tamaño de las células hemáticas, es decir es un comportamiento fisiológico. Los recuentos de referencia específicos para terneras desde el nacimiento hasta los 6 meses de edad son necesarios para RBC, VCM, CHCM, PLT y LYM (Brun-Hansen et al., 2006).

\subsection{Valores descriptivos de hematología}

En la Tabla 1 se advierten los valores hematológicos, mismos que se encuentran dentro de los distintos intervalos considerados como normales, de acuerdo con la bibliografía citada. En efecto, la edad mínima reportada al momento de tomar las muestras es el mismo día del nacimiento de la ternera (0 días), no obstante, la edad promedio en que se tomó esta medida es de 3.30 meses (99.11 días) con una desviación estándar de 1.99 meses (59.75 días), mientras que la edad máxima cumplida es de 6 meses (180 días). El número de muestras obtenidas fue de 283 , sin embargo, en mediciones únicamente de plaquetas (PLT), el equipo empleado no reportó valores para diez muestras; efecto que puede ser justificado por 
la posible formación de micro coágulos, ya que esto produce aglomeración de plaquetas, según señala Meyer \& Harvey (2000) y Villiers \& Blackwood (2013); por tal razón N en este caso fue de 273.

Tabla 8 Valores descriptivos de edad y valores hematológicos

\begin{tabular}{cccccc}
\hline & $\mathrm{N}$ & Mínimo & Máximo & Media & Desviación estándar \\
\hline Edad en días & 283 & 0.00 & 180.00 & 99.11 & 59.75 \\
RBC $10^{12} / \mathrm{L}$ & 283 & 0.70 & 7.44 & 4.96 & 0.81 \\
$\mathrm{HGB} \mathrm{g/L}$ & 283 & 57.00 & 128.00 & 98.99 & 11.57 \\
$\mathrm{HCT} \%$ & 283 & 3.30 & 41.20 & 25.17 & 4.42 \\
$\mathrm{VCM} \mathrm{fL}$ & 283 & 46.10 & 61.60 & 50.80 & 2.55 \\
$\mathrm{HCM} \mathrm{pg}$ & 283 & 14.50 & 88.70 & 20.75 & 8.23 \\
$\mathrm{CHCM} \mathrm{g/L}$ & 283 & 173.00 & 791.00 & 389.53 & 63.37 \\
$\mathrm{WBC} 10^{9} / \mathrm{L}$ & 283 & 1.80 & 42.40 & 12.42 & 5.40 \\
LYM $10^{9} / \mathrm{L}$ & 283 & 1.10 & 29.10 & 8.35 & 4.10 \\
MID $10^{9} / \mathrm{L}$ & 283 & 0.07 & 2.50 & 0.78 & 0.27 \\
GRAN $10^{9} / \mathrm{L}$ & 283 & 0.50 & 10.20 & 3.20 & 1.43 \\
PLT $10^{9} / \mathrm{L}$ & 273 & 41 & 875.00 & 261.19 & 110.13 \\
\hline
\end{tabular}

Los valores en recuento de glóbulos rojos (RBC) dio una media de $4.96 \times 10^{12} / \mathrm{L}$, en comparación con los intervalos de referencia de adultos señalados por Nuñez \& Bouda (2007): 5 a 8; Brun-Hansen et al. (2006): 4.6 a 6.9; Calzada et al. (2002): 5 a 10; Roldán, Luna \& Gasparotti (2006): $7.43 \pm 0.39$ / 7.05 \pm 0.33 en vacas en gestación y $6.98 \pm 0.60 / 6.95 \pm 0.46$ en vacas en lactancia en dos zonas diferentes; podemos ver que los valores son más altos que los encontrados y esto tiene relación con la edad de los animales, los pisos altitudinales y el tamaño de las células rojas, mismas que se encuentran más grandes en zonas altas y que se demuestra con el valor del VCM incrementado, es decir se produce una macrocitocis según manifestaron Barrios, Sandoval, Fernández, Camacaro, \& Sánchez (2011). De igual manera, Ježek et al. (2011) señalaron que en los terneros que reciben predominantemente leche, los valores de RBC disminuyen y los terneros se vuelven anémicos debido a la deficiencia de hierro. Arango, Oquendo, \& Agudelo (1992), obtuvieron un promedio de 3.1 en vaquillas de 6 a 12 meses, en un estudio realizado a una altitud de $2,400 \mathrm{msnm}$, lo que demuestra que los valores obtenidos no están lejos de la realidad expresada por estos investigadores.

La concentración de HGB se mantuvo constante, y los valores para la mayoría de las terneras cayeron dentro del intervalo de referencia para el ganado adulto, durante todo el período, en donde se encontró una media de $98.99 \mathrm{~g} / \mathrm{L}$, misma que está dentro de los rangos para animales jóvenes y adultos en diferentes pisos altitudinales (García, 2016; Brun-Hansen et al., 2006; Valarezo \& Jiménez, 1992; Arango et al., 1992) y bajo procesos de infección natural con nematodos como lo señala Barrios et al. (2013). Ježek et al. (2011) indicaron que el intervalo de referencia del adulto podría usarse para las mediciones de $\mathrm{HGB}$, para evaluar la masa de RBC en terneras, desde la primera semana hasta los 6 meses de edad, cuando las concentraciones de HGB se encuentran dentro de los rangos de referencia.

Analizando los valores de hematocrito $\left(\mathrm{HCT}^{3}\right)$, se obtuvo una media de $25.17 \%$, misma que se encuentra entre los valores mínimos permitidos, es así como Calzada et al. (2002) señalaron como valor mínimo $24 \%$ en animales adultos, valores que son utilizados en el Departamento de Patología ClínicaColegio de Médicos Veterinarios, UNAM. Además, estos valores están en relación con el RCB, ya que los terneros que son amamantados tienen deficiencia de hierro. Por otro lado, en un estudio realizado en Chile por Peede (1997), se encontró que la inclusión de probióticos en la dieta disminuye los valores de VGA en novillos, corroborando los hallazgos realizados por otros autores (Carraud \& Frencla, 1993; Villouta \& Rubio, 1978) quienes al observar el descenso de los valores de VGA, desde el primer mes hasta estabilizarse aproximadamente a los 4-6 meses de vida, lo atribuyeron a un componente mediado por la edad, ya que el número de eritrocitos disminuye progresivamente en forma paralela a un leve aumento de tamaño, hasta la madurez del individuo, confirmando lo anterior los valores de VGA obtenidos en este ensayo, y siguiendo su descenso hasta incluso después del séptimo mes de vida.

\footnotetext{
3 También conocido como VPC o VGA.
} 
Al hacer referencia a los índices eritrocíticos (VCM, HCM y CHCM) como medios de diagnóstico diferencial de los estados anémicos, Meyer \& Harvey (2000) señalaron que el VCM que representa el volumen promedio de un eritrocito solitario expresado e, $n$ fentolitros ${ }^{4}$, es el de mayor utilidad y su medición debe ser directa, es decir, en contadores celulares electrónicos. Por lo tanto, en el presente estudio tenemos una media de $50.80 \mathrm{fL}$ con valores mínimo de 46.10 y máximo de $61.60 ;$ los mismos que son superiores a los valores señalados en la bibliografía; esto nos lleva a ratificar de que en pisos altitudinales altos se produce una macrocitosis que tiene relación con la disminución de glóbulos rojos.

Los leucocitos (WBC) son células sanguíneas que cambian sus valores considerablemente después del nacimiento, por esto, en las primeras etapas de vida encontramos valores sumamente altos, que se consideran como una leucocitosis fisiológica en terneros (Benjamín, 1991). Ježek, et al. (2011) también señalan que el incremento en el número de leucocitos en terneros podría estar relacionado con el desarrollo del sistema inmune y la reacción a los nuevos antígenos, en algunos casos también a enfermedades subclínicas y son más altos que el intervalo de referencia para animales adultos. Los valores de WBC en nuestro estudio son más altos que los resultados establecidos por Mohri et al. (2007), Delgado et al. (2014), Calzada et al. (2002), Ježek et al. (2011), Nuñez \& Bouda (2007), Meyer \& Harvey (2000), Arango et al. (1992), y Valarezo \& Jiménez (1992). De la misma manera en el presente estudio se encontró mayor cantidad de linfocitos frente a los granulocitos, dando concordancia con lo que expresa (Meyer \& Harvey, 2000), quienes sostienen que los linfocitos por lo regular son más abundantes en porcinos, bovinos, ovinos y caprinos, mientras que perros, gatos y caballos tienen más neutrófilos.

La dinámica del número de PLT, en referencia a la edad de las terneras, fue similar a los resultados establecidos por Brun-Hansen et al. (2006), Ježek et al. (2011) y Cortez \& Noguera (2006) en relación estricta a animales jóvenes. De igual manera, en referencia de animales adultos, existe concordancia ya que los límites van desde 100 a 800 10/L (Nuñez \& Bouda, 2007).

\subsection{Correlación con la edad de los terneros}

$\mathrm{Al}$ evaluar la relación entre la edad y los valores hematológicos, expuesta en la Tabla 2, se advirtió que casi todos ellos presentan correlación, con excepción de RBC, HGB, MID y PLT. Valores hematológicos de HCT, VCM, WBC y LYM muestran correlación inversa, lo que significa que a medida que aumentan la edad, disminuyen sus valores. Por su parte, HCM, CHCM, MID y GRAN muestran correlaciones directas, lo que significa que mientras más edad aumentan sus valores. Es decir, están dentro de los procesos fisiológicos.

La mayoría de las correlaciones evidenciadas presenta una asociación baja con la edad, sin embargo, el VCM, que representa el volumen promedio de un eritrocito solitario, expresado en fentolitros, tiene una asociación media $\left(-0.531^{* *}, \mathrm{R}^{2}=0.28\right)$, misma que disminuye a medida que aumenta la edad de las terneras. Esto corrobora con lo que manifiesta Brun-Hansen et al. (2006) en su investigación en Noruega que dice: "El VCM estaba justo debajo del intervalo de referencia adulto durante la primera semana de vida, y luego disminuyó constantemente a un mínimo de $30 \mathrm{fL}$ a las 2325 semanas. Se ha descrito anteriormente una disminución en el VCM y se refiere a la sustitución de glóbulos rojos que contienen hemoglobina fetal con glóbulos rojos más pequeños que contienen hemoglobina. El VCM inferior se compensa con el mayor número de glóbulos rojos para mantener una concentración normal de HGB".

Tabla 9. Correlación $r$ de Pearson entre los valores hematológicos, VCM y edad.

\begin{tabular}{lrrrrrrrrrrrr}
\hline & Días & VCM & RBC & HGB & HCT & HCM & CHCM & WBC & LYM\# & MID\# & GRAN\# & PLT \\
\hline Días & 1 & $-.531^{* *}$ & -.100 & .013 & $-.244^{* *}$ & $.152^{*}$ & $.119^{*}$ & $-.251^{* *}$ & $-.210^{* *}$ & -.094 & $-.249^{* *}$ & -.089 \\
VCM & & 1 & $.176^{* *}$ & $.176^{* *}$ & $.450^{* *}$ & -.091 & $-.247^{* *}$ & $.306^{* *}$ & $.317^{* *}$ & .109 & $.162^{* *}$ & .074 \\
\hline
\end{tabular}

Leyenda: $\#=10^{9} / \mathrm{L}, * *=\mathrm{p} \leq 0.01,{ }^{*}=\mathrm{p} \leq 0.05$

\footnotetext{
${ }^{4}$ Fentolitro (fL o fl): unidade de volume igual a $10^{-15}$ litro, equivale a $1 \mu \mathrm{m}^{3}$
} 


\section{CONCLUSIONES}

En el presente estudio se revelan variaciones ontogénicas de varios parámetros y se establecieron medias y correlaciones entre los valores hematológicos de las terneras, a la vez se puede advertir que casi todos ellos presentan correlación, con excepción de RBC, HGB, MID y PLT. Mohri et al. (2007) señalan que los valores de referencia específicos de la edad deben considerarse para la interpretación precisa de los resultados de laboratorio. Se debe tomar en consideración que en los terneros que reciben predominantemente leche como fuente nutricional, los valores de RBC disminuyen, y, los terneros se vuelven anémicos debido a la deficiencia de hierro, de igual manera cabe destacar que por un proceso fisiológico, en pisos altitudinales altos se produce una macrocitosis que tiene relación con la disminución de glóbulos rojos. Debido a que los animales investigados provienen de un hato lechero, los valores son más representativos para terneras que van a ser destinadas como remplazo en el hato, sim embargo, estos valores podrían también servir como referencia para otras razas de bovinos.

\section{REFERENCIAS}

Arango, N. C., Oquendo, R., Agudelo, G. L. (1992). Influencia de la altitud en parámetros fisiológicos generales y heméticos de bovinos Holstein. Medellin, Colombia: Revista Facultad Nacional de Agronomía, 45(2), 51-60.

Barrios, M., Sandoval, E., Fernández, D., Camacaro, O., Sánchez, D. (2011). Diagnóstico y evaluación de tratamientos de la anemia macrocítica en becerros. Yaracuy, Venezuela: Redvet, 12(7), 1-11.

Barrios, M., Sandoval, E., Borges, J., Sánchez, D., Rondón, Y., Márquez, O., Dávila, L. (2013). Perfil leucocitario en becerros anémicos infectados naturalmente con nematodos gastrointestinales. Maracay, Venezuela: Zootecnia Tropical, 31(3).

Benjamín, M. M. (1991). Manual de patología clínica en veterinaria. México D.F., México: Editorial LIMUSA.

Brun-Hansen, H., Kampen, A., Lund, A. (2006). Hematologic values in calves during the first 6 months of life. Veterinary Clinical Pathology, 35(2), 182-187.

Calzada, P., Morales, E., Quiroz-Rocha, G., Salmerón, F., García, C., Hernández, J. (2002). Valores hematológicos en vacas de raza Holstein-Friesian seropositivas a Neospora caninum de la cuenca lechera de Tizayuca, Hidalgo, México. Veterinaria México, 33(2), 119-124.

Carraud, A., Frencla, J. P. (1993). Fattening of beef cattle. Relation between blood values and carcass quality. Effect of the addition of a yeast probiotic on these values. Bulletin des G.T.V., 3, 11-27.

Coppo, J. A. (2010). Interpretación de análisis clínicos en perros y gatos. 372 p. Salta, Argentina: EUCASA Ediciones, Universidad Católica de Salta.

Coppo, J. A., Mussart, N. B. (2006). Evolución de parámetros hemáticos de terneros media sangre cebú en crecimiento. Revista del Instituto Agrotécnico de la UNNE, Chaco, Argentina, Agrotecnia, 16, 5-11.

Cortez, M. J., Noguera, A. J. (2006). Estudio hematológico y parasitológico como apoyo al diagnóstico de las alteraciones metabólicas en terneros de 1 a 6 meses de edad. Tesis (Lic. en Medicina Veterinaria), Universidad Nacional Autónoma de Nicaragua, León. 72 p. Disponible en http://riul.unanleon.edu.ni:8080/jspui/bitstream/ 123456789/1022/1/199974.pdf.

Delgado, R., Rodríguez, H., Barreto, G., Vazquez, R. (2014). Efecto probiótico de Saccharomyces cerevisiae en parámetros hemáticos y metabólicos de terneros en pastoreo. Revista de Producción Animal, 26(3), 2010-2015.

García, M. S. (2016). Efecto de la levadura Saccharomyces cerevisiae en condición corporal, alzada, ganancia diaria de peso, parámetros hematológicos y metabólicos con terneros de remplazo criados al pastoreo en la Hacienda Nero. 76 p. Tesis pregrado de Médica Veterinaria y 
Zootecnista, Universidad de Cuenca, Cuenca, Ecuador. Disponible en http://dspace.ucuenca.edu.ec/bitstream/123456789/25292/1/Tesis.pdf.pdf

Ježek, J., Nemec, M., Starič, J., Klinkon, M. (2011). Age related changes and reference intervals of haematological variables in dairy calves. Bulletin of the Veterinary Institute in Pulawy, 55(3), 471-478.

Klein, B. (2014). Fisiología veterinaria. Barcelona, España: Publ. Elsevier.

Latimer, K., Mahaffey, E., Prasse, K. (2005). Patología clínica veterinaria. Barcelona, España: Multimédica.

Meyer, D. J., Harvey, J. W. (2000). El laboratorio en medicina veterinaria: Interpretación y diagnóstico. 397 p. Buenos Aires, Argentina: Publ. Inter-Médica.

Mohri, M., Sharifi, K., Eidi, S. (2007). Hematology and serum biochemistry of Holstein dairy calves: Age related changes and comparison with blood composition in adults. Research in Veterinary Science, 83(1), 30-39. https://doi.org/10.1016/j.rvsc.2006.10.017

Nuñez, L., Bouda, J. (2007). Patología clínica veterinaria. México D.F., México: UNAM-FMVZ.

Paez, P. A., Campos, R., Patiño, L. (2013). Suplementación y metabolitos de hierro en neonatos bovinos, en condiciones de trópico. Acta Agronómica, 62(1), 59-65.

Peede, M. L. (1997). Efecto del probiotico Bovex® en la ganancia de peso y composición sanguínea de terneros de lechería. Tesis de grado, 59 p. Escuela de Medicina Veterinaria, Facultad de Ciencias Veterinarias, Universidad Austral de Chile. Disponible en http://cybertesis.uach.cl/tesis/uach/1997/fvp373e/doc/fvp373e.pdf

Reece, W. O. (2004). Dukes Fisiología de los animales domésticos. 31 p. Zaragoza, España: Acriba.

Roldán, V. P., Gasparotti, M. L., Luna, M., Piérola, F., Sola, J. M., Gapel, C., Pinto, M. (2005). Análisis del perfil hematológico de vacas gestantes de la región centro de Santa Fe. Revista Electrónica de Veterinaria REDVET, VI(12), 1-4.

Roldán, V. P., Luna, M. L., Gasparotti, M. L. (2006). Variaciones en perfiles hematológico de bovinos lecheros de la Cuenca del salado en distintos estados fisiológicos. Revista Electrónica de Veterinaria REDVET, VII(12), 1-4.

Saíz, A. A., Antuna Bizarro, S., Anzaldúa Arce, S. R. (2010). Fisiología veterinaria e introducción a la fisiología de los procesos productivos. 347 p. México D.F., México: Universidad Nacional Autónoma de México.

Valarezo, P. L., Jiménez, E. G. (1992). Determinación de valores hematológicos en bovinos aparentemente sanos en las parroquias: El Valle, Nulti y San Joaquín, provincia del Azuay. Tesis de pregrado, 90 p. Facultad de Ciencias Agropecuaria, Universidad de Cuenca. Obtenido de http://dspace.ucuenca.edu.ec/handle/123456789/19958

Villiers, E., Blackwood, L. (2013). Manual de diagnóstico de laboratorio en pequeños animales. ( $2^{\mathrm{a}}$ ed.). Barcelona, España: Ediciones S. 648 p.

Villouta, G., Rubio, T. (1978). Valores hematológicos en terneros Holstein Frisian de 3 a 180 días de edad. Archivos de Medicina Veterinaria, 10(1), 22-26. 